\title{
Modeling and Simulation of Triple Coupled Cantilever Sensor for Mass Sensing Applications
}

\author{
Nalluri Siddaiah $^{\# \$}$, D.V. Rama koti Reddy ${ }^{\$}$, Y Bhavani Sankar ${ }^{\#}$, R. Anil Kumar \\ Hossein Pakdast* \\ \#Department of Electronics \& Communication Engineering, K L University, Vaddeswaram \\ Guntur (dt), Andhra Pradesh, India \\ ${ }^{\$}$ Department of Instrumentation Engineering, College of Engineering, Andhra University, Andhra Pradesh, India \\ *Laboratorio TASC, IOM, CNR, Trieste, Italy
}

\begin{abstract}
Article Info
Article history:

Received Nov 6, 2014

Revised Feb 23, 2015

Accepted Mar 10, 2015

Keyword:

Finite element analysis

Mass detection

Micro-cantilever

ABSTRACT

Cantilever sensors have been the growing attention in last decades and their use as a mass detector. This work presents design, modeling and analysis of Triple coupled cantilever (TCC) sensor using MEMS simulation software Comsol Multiphysics with critical dimensions of $100 \mu \mathrm{m}$ length, $20 \mu \mathrm{m}$ width and $2 \mu \mathrm{m}$ thickness. Simulations were performed based on finite element modeling techniques, where different resonant frequencies were observed for different modes of operation. It is also observed that the resonant frequency of the sensor decreases as some mass is applied on one particular cantilever. The various parameters greatly affecting the performance of TCC such as resonant frequency, dimensions, material and pressure or force applied on it. We also observed that while adding some mass on any one lateral cantilever, the resonant frequency of that respective mode reduced.
\end{abstract}

Sensor

Triple coupled cantilever
Copyright (C) 2015 Institute of Advanced Engineering and Science. All rights reserved.

\section{Corresponding Author:}

Nalluri Siddaiah,

Department of Electronics \& Communication Engineering,

K L University, Vaddeswaram,

Andhra Pradesh, India

Email: nalluri.siddu@kluniversity.in

\section{INTRODUCTION}

The widespread availability of inexpensive microfabricated cantilever structures has resulted in renewed interest based on surface stress cantilever sensors.Many microcantilever sensors which build on the measurement of displacement and smallest detectable motion have been utilized for high precision chemical and small force detection. The field of microcantilever sensors has been very active in recent years. The most applications are found in the study of physisorptions, chemisorptions and bimolecular interaction analysis. Research progress in cantilever sensors under different environments has provided new dimensions of complex biochemical reactions as hybridization of DNA or pathogen detection in antibody-antigen functionalization and proteomics.

Mechanically Triple coupled cantilevers sensors offers several advantages over single cantilever sensors including less vacuum requirements for operation, mass localization, insensitivity to surface stress and distribution to a specific adsorbtion. Spletzer et.al proposed coupled cantilevers for mode localization derived mass detection [1]. B. Ilic et.al proposed, resonant microstructure and resonant microcantilevers as very sensitive tools for mass detection down to single molecule level [2]. With the invention of Atomic force microscope (AFM) in 1986 the use of microcantilever resonators became very popular in mass detection. A large variety of sensor technologies based on cantilever structures were applied as mass detectors such as biological, physical, chemical, medical diagnostics and environmental monitoring $[3,4,5,6]$. 
On the other side of coin, from dimension point of view the challenge lie in cantilever optimization towards its sensitivity limit of detecting individual molecules using nanomechanical detection ranges in hybridization, proteomics, mass sensing applications. Each cantilever is modeled as a damped simple harmonic oscillator, while the effect of the overhang coupling is modeled as a spring connecting three oscillators. The effective mass of analyte in bimolecular mass detection being detected by using resonant frequency shift, between before and after analyte interaction and stiffness coefficient. Ideally coupled cantilevers are identical, however manufacturing tolerances and material variations cause the properties of one cantilever to differ slightly from another.

\subsection{Triple coupled cantilever}

To design TCC, first individual cantilevers was designed with critical dimensions of $100 \mu \mathrm{m}$ length, $20 \mu \mathrm{m}$ width and $2 \mu \mathrm{m}$ thickness. After that all these three individual cantilevers were coupled with overhang dimensions of $100 \mu \mathrm{m}$ long, $20 \mu \mathrm{m}$ width and $2 \mu \mathrm{m}$. One side of overhang boundaries are set to be fixed and remaining all boundaries are set to be free. Here we used different materials for all subdomains of TCC sensor and we got different modes by simulation.

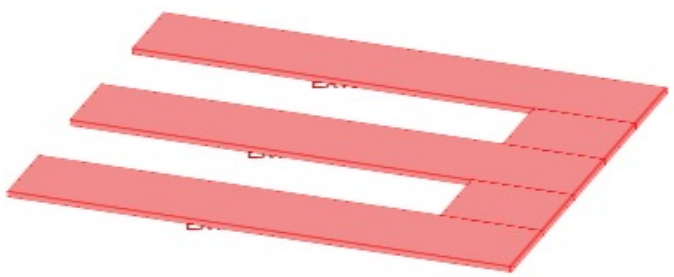

Figure 1. Schematic diagram of Triple coupled cantilever (TCC)

\subsection{Finite Element Analysis}

Meshing: Finite element analysis is a numerical solution of the field problems where mathematically a field problem is described by differential equation or by integral expression. These finite elements are characterized for a specific pieces of small size structure, which have a simple variation, explained by polynomial (rectangular, quadrilateral, triangular, tetrahedral \& Manhattan) are spanned by element to element connected by nodes. This assembly gives the FEA of the entire structure where it is referred as Mesh. [Robert. et.al]. A TCC is applied with meshing (tetrahedral).

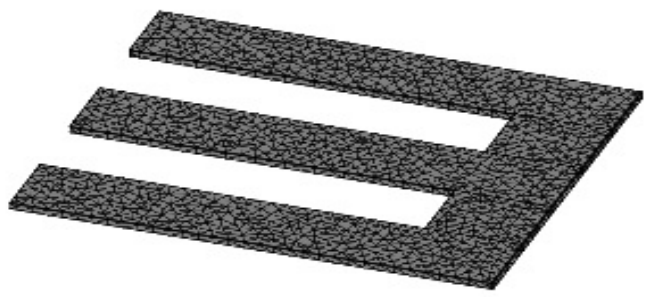

Figure 2. Meshing schematic of TCC

\section{MODELING AND SIMULATION RESULTS}

The TCC is modeled for different materials like Semiconductors, insulators and polymers and corresponding resonant frequencies are tabulated in tablel. The dimensions of TCC are unaltered \& simulation is verified for the behavior of resonant frequency which corresponds to material properties like young's modulus and density. Verification of these FEA of the structure yields to close adaptation of materials which are biocompatible and Bio-degradable, signature properties for biological application. The relation between the resonant frequencies to Young's Modulus \& Density are plotted where a merely optimized material for the specified Young's Modulus and density are used for fabrication. As BioMEMS is a wide collateral field where the material science show a prominent importance for individual application. TCC with Semiconductors (Si, Poly-Si), Insulators (SiC, Si3N4) and polymers (PTFE, PMMA) which given a close interpretation for the material selection in Bio Sensing applications. 

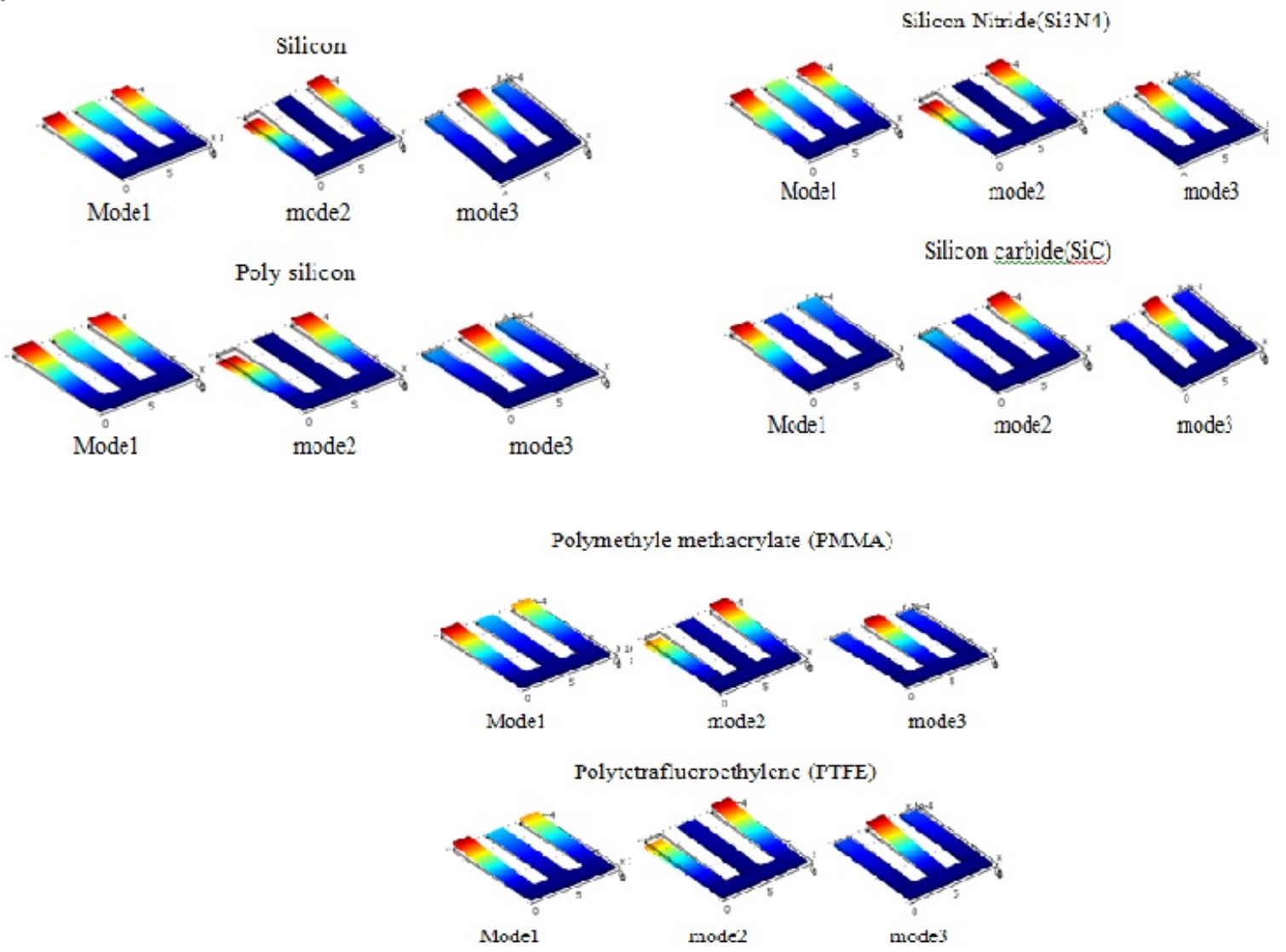

Mode 1

From above simulation results in mode1 for all types of materials three cantilevers in TCC are vibrated in same phase with different resonant frequencies. It is observed that Silicon carbide (SiC) materiel posses high resonant frequency $(366.474 \mathrm{Khz})$. It is also observed that Ploytrtrafluorethylene (PTFE) polymer material have lowest resonant frequency $(10.146 \mathrm{Khz})$.

Mode 2

In mode 2 The TCC two lateral cantilevers are vibrated in opposite phase but central cantilever is in stationary. In this mode also SiC materiel having highest resonant frequency and PTFE has lowest resonant frequency. This mode is very useful to detect the single molecules, viruses, bacteria etc.

Mode 3

In mode 3 two lateral cantilevers of TCC are vibrated in same phase but central cantilever is vibrated is in opposite phase with lateral cantilevers. In this also $\mathrm{SiC}$ material has highest resonant frequency and PTFE material has lowest frequency.

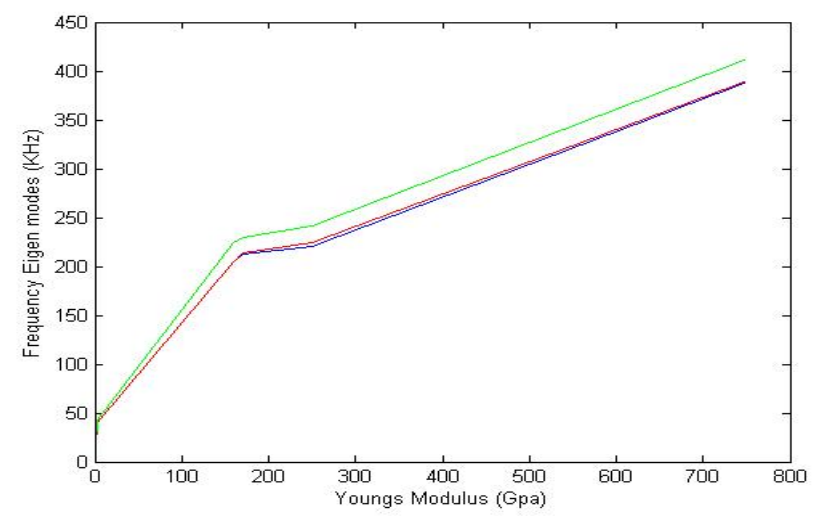

Figure 3. Eignen frequency Vs Young Modulus 


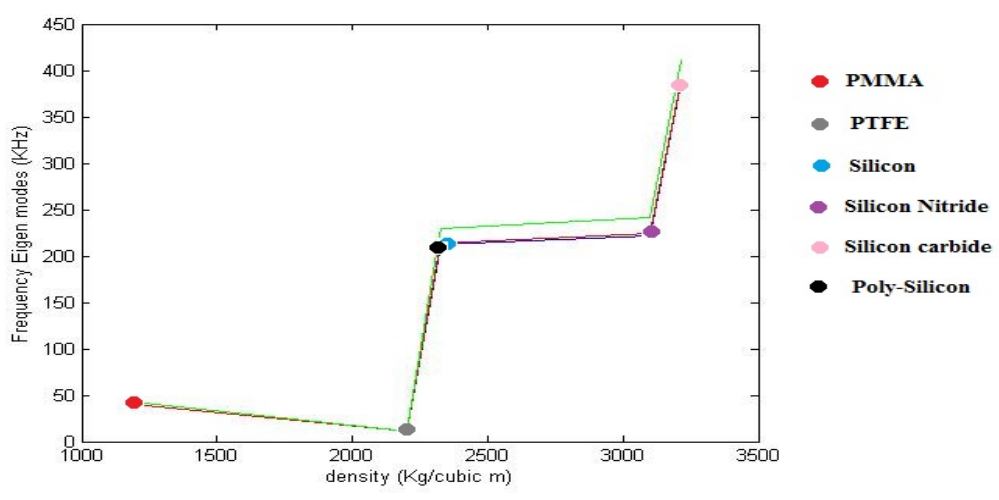

Figure 3. Eignen frequency Vs Young Modulus

\subsection{Fibroblast Detection}

A cell that synthesizes the extracellular matrix a collagen which plays a crucial role in wound healing, sp called as connective tissue. These are morphologically heterogeneous with diverse appearances depending on location \& activity. The main functionality is to organize the structural integrity of tissue and their connectivity. They do carry out the functionality by continuous secreting precursors liquids of extracellular matrix which are extrafibrillar matrix (water, hyaluroa, glycoprotiens) and variety of fibers. This composition determines the properties of connective tissues where the mitosis is triggered by the fibrocyctes upon the tissue damage. This can be termed as (hFGF) termed as human fibroblast $[7,8]$ which are produced by epithelial, tumor \& other cell types. It is used as a factor for cultivating embryonic stem cells in culture without inducing differentiation. It shows high affinity for heapran sulfate [7] [8]. Widely employed approaches in the synthesis of blood compatible materials are heparinization, in which synthetic polymers are coated or immobilized with heparins [9-12]. Heparin (18.2 mg) 4-azidoaniline (7.75 mg), and WSC (10 mg) were dissolved in deionized water $(20 \mathrm{~mL}) \& \mathrm{pH}$ is ms maintained to be 7 . The solution was stirred at $4 \mathrm{C}$ for $24 \mathrm{hr}$ and ultra filtrated, the residue was repeatedly washed with distilled water for the removal of 4azidoaniline. This micro pattern -immobilization can be carried out using Dip Pen Nanolithography (DPN), capillary system or Nanoenabler on lateral cantilever of TCC. Enzyme functionalized TCC can be used to study its frequency shift for the growth of fibroblast [21].

\subsection{Glucose Sensor}

Monitoring Glucose in diabetic patients is very much essential; here we report a novel technique for micromechanical detection of biologically relevant glucose by immobilization of glucose oxidase (GOx) onto a surface of lateral cantilever of Triple Couple Cantilever (TCC). In this paper we propose a novel glucose biosensor based on enzyme specificity to fabricate a highly selective glucose biosensor. The enzyme functionalized TCC under goes frequency shift due to the reactions between glucose and GOx immobilized TCC.

Molecular adsorption, when confined to one surface of TCC, results in differential surface stress that leads to shift in frequency. General applications of this label-free detection method have been shown for DNA hybridization, for single -base mismatches detection [13, 14] and nano- mechanical motion induced by antibody- antigen reaction $[15,16]$.

Diabetes mellitus is a disease in which cells fails to take glucose due to either lack of insulin or an insensitivity to insulin, the associates levels of blood glucose for prolonged periods leads to different side effects, including retinopathy, nephropathy, neuropathy and heart related issues. To stabilize the blood glucose levels is major criteria to delay the onset of diabetes progression $[18,19]$. Glucose detection was achieved by immobilizing a layer of glucose oxidase on the surface of the lateral cantilevers of TCC and then detecting frequency shift between before and after GOx Surface modification of TCC \& upon detection of glucose.

In this detection scheme we will use TCC dimensions of 100um long, 20um wide and 2um thickness.

Functionalization of Glucose Oxidase (GOx) onto the surface of lateral cantilevers of TCC by crosses linking with Glutaraldehyde (GA) in the presence of Bovine serum albumin (BSA) [20]. The enzyme 
solution is prepared by dissolving $20 \mathrm{mg}$ of GOx, $5 \mathrm{mg}$ of BSA and $40 \mathrm{uL}$ of $50 \%$ GA in sequence in $1 \mathrm{~mL}$ of buffer solution $(\mathrm{PH}=7)$. [14] A well defined capillary system or Dip pen nanolithography (DPN) or Nanoenabler is used to dispense it onto the lateral cantilever of TCC. Enzyme-functionalized TCC can be used to study its frequency shift response to various concentrations of glucose.

The responses and non linearity of the micro cantilever are studied under different parameter conditions, applied voltages and various gaps between capacitor plates. The Non linear dynamical behaviour are characterised by using phase portrait and point care mapping in phase space. The rational parameters the analytical solutions and numerical simulations are similar. Basically MEMS devices are inherently nonlinear. Some nonlinear mechanical characteristics are large deformations, surface contact creep phenomena and damping effects. Many Researchers have investigated these characteristics of MEMS under various load conditions.

Most MEMS devices are non-linear and micro-scale effects and coupled fields give rise to the complete non-linearities, mechanical-deformation, surface contact, time dependent masses and non-linear damping effects etc. [15], damping like squeeze film damping.All these non-linearities play a major role in corresponding characterisation of MEMS structures. In case of MEMS where dealing with biological entities and these are countable since the excitation or actuation of cantilever structures is generally electrostatic [16] where direct current is superimposed to an alternate current harmonic -voltage. These actuation methods again add hassle to calibration with hysteresis and dynamic instabilities [17]. So in order to minimise all these instabilities and non-linearities of the micro devices the dynamic excitation of the TCC is carried out using LDV laser doppler vibrometer where the excitation is carried use a pulsed blue laser and the shift in resonant frequency is calibrated using the shift in red laser which is incident of the dynamic excited cantilever with phase shift of the laser beam the shift in resonant frequency can be calibrated. This technique holds good for biological entities where the signal is very small and easily affected by the external disturbances. A small change in the biological parameter counts a lot more difference in calibration end because the output voltage ranges will be in pico-micro and change is in few less than that. In such order the Dynamic mode measurement of the cantilever using vibrometer yields a good result in characterising the functionalized cantilever with biological entities. In our experiment the fibroblast and glucose are sensed using functionalization on the TCC.

\section{EXPERIMENTAL SETUP}

The TCC is mounted in a measurement chamber by maintaining a vaccum, excited by a frequency modulated red laser [7], detected using a green laser \& four quadrant photodiode (4QPC). A manual micropositioning xy stage is used to move the whole chip with respect to the optical path to record the frequency of each cantilever by moving second micro-positioning xy stage to maximize the signal on 4QPC. We first measure the frequency of middle cantilever in second mode for the stabilizing and finding out the nonlinearity among the cantilever in fabrication. Upon surface immobilization of GOx on lateral cantilever with which the frequency of second cantilever is measured. Before characterizing this each device is characterized in second mode for set-up alignment of the TCC which distracts the readings. If the amplitude values are affected equally can be ordered as misalignment resulting in amplitude ratio of $\rho_{\mathrm{a}}$. With glucose in detection where the glucose molecules binds to GOx on the surface of the lateral cantilevers. The measurements are repeated with measuring bench. As added mass on lateral cantilevers of TCC effects the amplitude of the middle cantilever in second mode of operation. The shift in frequency is measured upon considering the $\rho_{\mathrm{a}}$ gives exact mass added. This can be measured by red \& green laser on photo detector. 


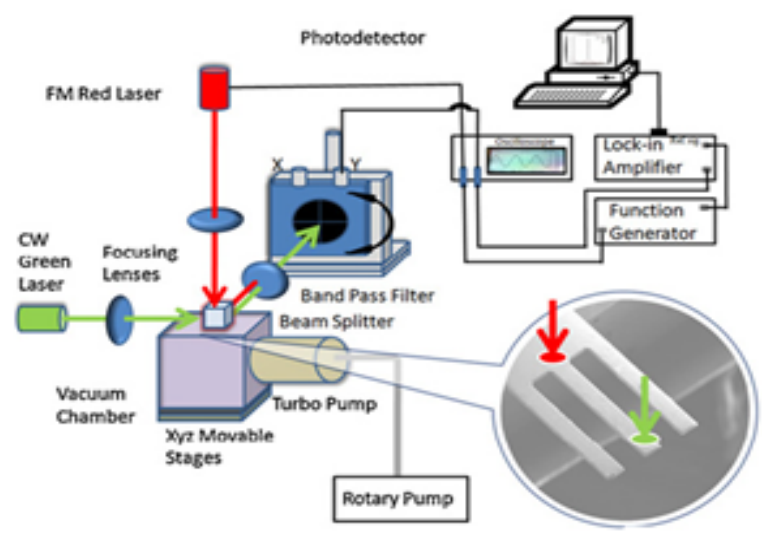

Figure 4. Experimental setup

\section{CONCLUSION}

The TCC is modeled for different materials and the resonant frequency of each Eigen mode is simulated and is observed to be using this as bio-sensor. The TCC is actuated using Dielectric force gradient method and Optical excitation for each corresponding Eigen state. A surface modification protocol is proposed for Fibroblast and Glucose [22] for TCC to use it as Bio-Sensor such that the work can further extended for different pathogens, or enzymes and Protein base sensors [23]. This can be further extended to DNA base bio-sensors where PCR and ELISA base tests can be done at Micro level using MEMS based Biosensors by using Triple couple cantilevers.

\section{REFERENCES}

[1] Hossein Pakdast, Marco Lazzarino, SNA -7633, Sensors and Actuators A, (2012).

[2] B. Ilic, Y. Yang, K. Aubin, R. Reichenbach, S. Krylov, H.G. Craighead, Enumeration of DNA molecules bound a nanomechanical oscillator, Nano Lett. 5 (2005) 254102.

[3] A. Qazi, D. Nonis, A. Pozzato, M. Tormen, M. Lazzarino, S. Carrato, G. Scoles, Asymmetrical twin cantilevers for single molecule detection, Appl. Phys. Lett. 90 (2007) 173118.

[4] M. Spletzer, A. Raman, A.Q. Wu, X. Xu, R. Reifenberger, Ultrasensitive mass sensing using mode localization in coupled microcantilevers, Appl. Phys. Lett. 88 (2006) 254102.

[5] E. Gil-Santos, D. Ramos, A. Jana, M. Calleja, A. Raman, J. Tamayo, Mass sensing basedondeterministic and stochastic responses of elastically coupled nanocantilevers, Nano Lett. (2009).

[6] H. Okamoto, T. Kamada, K. Onomitsu, I. Mahboob, H. Yamaguchi, Optical tuning of coupled micromechanical resonators, Appl. Phys. Express 2 (2009).

[7] Dvorak, P. and Hampl, A. (2005) Folia Histochem Cytobiol 43, 203-B.

[8] Ornitz, D.M. and Itoh, N. (2001) Genome Biol 2 Reviews 3005.

[9] Shi,Y.et.al(2008) CritRev Oncol Hematol 65,43-53.

[10] Fontijn, D. et.al. (2006 Re J Cancer 94 1627-36.

[11] Marek, L.et.al. (2009) Mol Pharmacol 75, 196-207.

[12] Acevedo, V.D. et.al. (2009) Cell Cycle 8, 580-8.

[13] K. M. Hanson, H. Ji, G. Wu, R. Datar, R. Cote, A. Majumdar and T. Thundat, Anal. Chem73, 1567, (2001).

[14] J. Fritz, M.K. Baller, H.P. Lang, H. Rothuizen, P. Vettiger, E. Meyer, H.J. Güntherodt, Ch. Gerberand J.K. Gimzewski, Science, 288, 316, (2000).

[15] Wenming Zhang, Guang Meng, Nonlinear dynamical system of micro-cantilever under combined parametric and forcing excitations in MEMS, Sensors and Actuators A, 119 (2005) 291-299.

[16] J. Chung, Dynamic analysis of a rotating cantilever beam by using the finite element method, Journal of Sound and vibration (2002) 249(1), 147\}164.

[17] Fadi M. Alsaleem, Mohammad I. Younis, Member, ASME, and Laura Ruzziconi, An Experimental and Theoretical Investigation of Dynamic Pull-In in MEMS Resonators Actuated Electrostatically, Journal Of Microelectromechanical Systems, Vol. 19, No. 4, August 2010.

[18] G. Wu, R. Datar, K. Hanson, T. Thundat, R. Cote and A. Majumdar, Nature Biotechnology, 19, 856, (2001).

[19] K. Stevenson, A. Mehta, P. Sachenko, K. Hanson and T. Thundat, Langmuir, 18, 8732, (2002).

[20] G. S. Wilson and Y. Hu, Chem. Rev, 100, 2693, (2000).

[21] J. Wang, Electroanal. 13, 983, (2001).

[22] Jianhong Pei, Fang Tian, and Thomas Thundat, Proc. vol.776, Materials Research Society.

[23] Yong Soon Park, Yoshihiro Ito, Micropattern-immoblization of heparin to regulate cell growth with fibroblast growth factor. Pg.no.117-122, Cytotechnology 2000. 